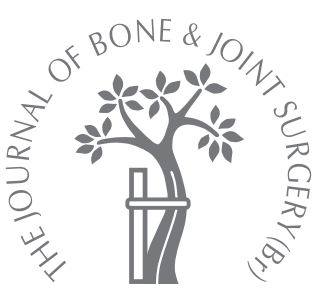

A. Kulkarni, P. Partington, D. Kelly, S. Muller,

From Wansbeck General Hospital, Ashington, England

\title{
Disc calibration for digital templating in hip replacement
}

Digital radiography is becoming widespread. Accurate pre-operative templating of digital images of the hip traditionally involves positioning a calibration object at its centre. This can be difficult and cause embarrassment. We have devised a method whereby a planar disc placed on the radiographic cassette accounts for the expected magnification. Initial examination of 50 pelvic CT scans showed a mean hip centre distance of $117 \mathrm{~mm}$ (79 to 142) above the gluteal skin. Further calculations predicted that a disc of $37.17 \mathbf{~ m m}$ diameter, placed on the cassette, would appear identical to a $30 \mathrm{~mm}$ sphere placed at the level of the centre of the hip as requested by our templating software. We assessed accuracy and reproducibility by 'reverse calibration' of 20 radiographs taken three months after hip replacement using simultaneous sphere and disc methods, and a further 20 with a precision disc of accurate size. Even when variations in patient size were ignored, the disc proved more accurate and reliable than the sphere.

The technique is reliable, robust, cost effective and acceptable to patients and radiographers. It can easily be used in any radiography department after a few simple calculations and manufacture of appropriately-sized discs.

Digital radiography and picture archiving and communication systems (PACS) are rapidly replacing conventional radiographs in the United Kingdom. ${ }^{1}$ Computed radiography allows the minimum correct exposure for a satisfactory image and digital radiography has largely displaced conventional film. Before PACS, acetate templates were used with conventional radiographs of the pelvis for planning hip replacement surgery.

Pre-operative templating allows assessment for minimal removal of bone, restoration of the centre of rotation, minimises malposition of the components and optimises limb length and femoral offset. ${ }^{2}$ In our hospital, digital radiographs and PACS have replaced conventional radiographs but variable magnification of printed digital images makes conventional templating inaccurate compared with analogue films. ${ }^{3,4}$

Accurate templating of digital radiographs can only be performed on correctly calibrated images. Using a British ten pence coin taped to the thigh at the level of the greater trochanter, Conn, Clarke and Hallett ${ }^{5}$ calculated the magnification of conventional analogue films and found the method accurate in 22 of 32 hips, an improvement from $59.5 \%$ to $68.8 \%$. Oddy et $\mathrm{al}^{6}$ used a ten pence coin in a similar way for correcting the magnification of digital radiographs. They com- pared on-screen imaging and hard-copy templating, using standard acetate templates. They found improved accuracy and reproducibility when templating adjusted images directly on-screen, compared with printed copy templating using acetate templates. Wimsey, Pickard and Shaw ${ }^{7}$ developed a scaling device using a Swiss five franc coin at the level of the greater trochanter. They compared these results with those obtained by measuring the distance between the antero-superior iliac spines with a caliper. They demonstrated an accuracy of $98.9 \%$ with the coin method, the maximum margin of error of which was $2.44 \%$, significantly better than the caliper method.

The weakness of these techniques is their requirement to assess accurately the height of the greater trochanter from the table and then to place and hold a calibration object at that position.

We used 'Mdesk' digital templating software (RSA Biomedical, Umea, Sweden), which recommends that a $30 \mathrm{~mm}$ sphere be placed between the patient's legs at the height of the centre of the hip. However, there are infectioncontrol concerns about reusable calibration objects placed in the perineum and embarrassment about putting them close to intimate areas. These make the technique unpopular with radiographers. 


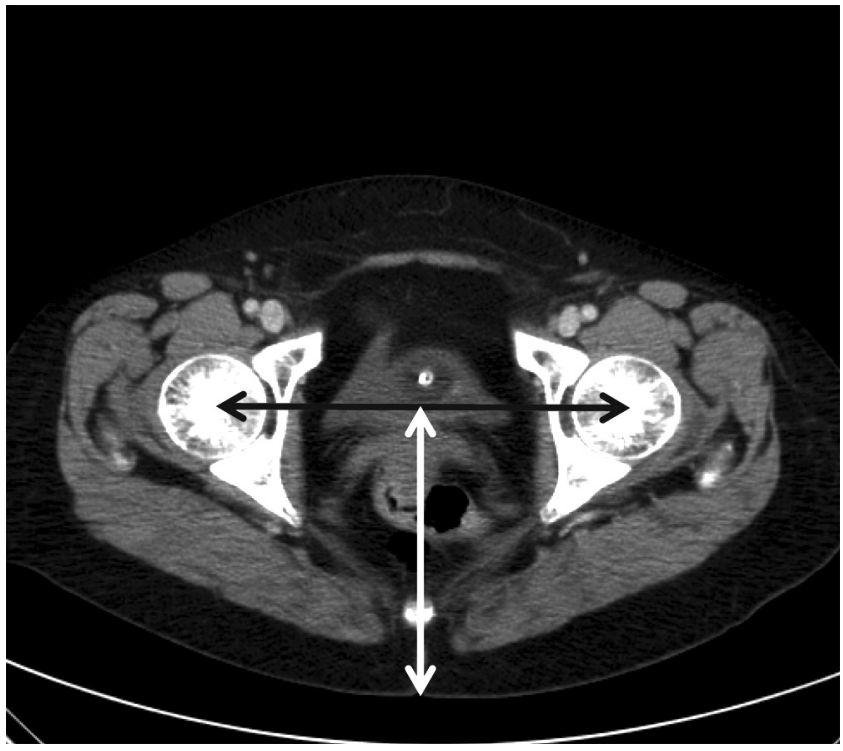

Fig. 1

CT measurement for hip-to-table distance. The black line connects hip centres and the grey line shows hip-to-table distance.

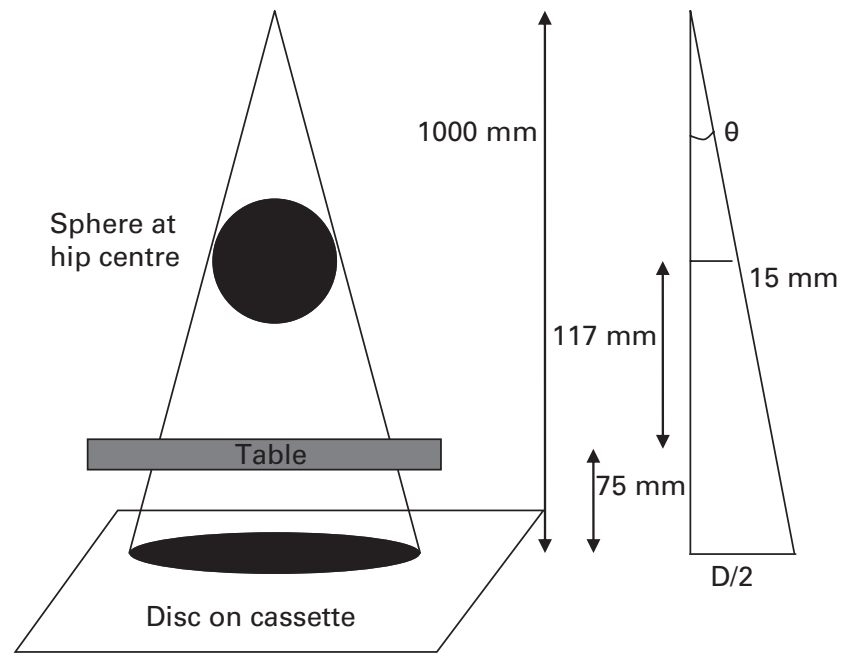

$$
\begin{aligned}
& (D / 2) / 1000=\tan \theta=15 /(1000-120-75) \\
& D / 2=151000 /(1000-195) \\
& D=37.17 \mathrm{~mm}
\end{aligned}
$$

Fig. 2

Diagram showing sphere and disc arrangement and calculations.

We attempted to resolve these problems with a simple, cheap, reliable and reproducible technique without the need to print inaccurate non-calibrated hard copies.

Using CT, we assessed the distance between the table and the centre of the hip to determine where calibration devices should be placed. We then developed a disc method accounting for magnification and, finally, we assessed the accuracy and reproducibility of the method by comparing its reliability with the manufacturer's technique of using a $30 \mathrm{~mm}$ sphere.

\section{Patients and Methods}

In order to determine the distance from the table to the hip centre, we randomly selected 50 abdominal/pelvic CT scans performed for non-orthopaedic indications. These images are calibrated automatically and subsequent measurements are accurate. The centre-to-table distance was the perpendicular distance from the table in the natal cleft to a transverse line between the centres of the two hips (Fig. 1). The first 20 scans were subject to independent measurement by two authors (AK and SM) and as agreement was extremely high (intraclass correlation coefficient with two-way analysis $=0.89$ ), the remaining 30 were measured by the first author (AK) only.

Mdesk software (RSA Biomedical) allows a single mouseclick calibration of the image using edge-detection techniques to identify the projected edge of a $30 \mathrm{~mm}$ sphere. The software can be fooled into calibrating for a $30 \mathrm{~mm}$ sphere at the level of the centre of the hip by placing a disc of appropriate diameter directly on and in the plane of the cassette. The subsequent image of the disc then appears identical to a sphere at the level of the hip. Wherever the disc is placed on the cassette it generates a circular image without parallax distortion, as may occur with a coin placed off-centre above the cassette. The method therefore allows the Mdesk software to calibrate an apparent sphere.

Trigonometric calculations were used to determine the appropriate disc size (Fig. 2). The CT measurements recorded the previously provided hip-to-table distance and the table specification gave the table-to-cassette distance.

Our table had a table-to-cassette distance of $75 \mathrm{~mm}$. The CT measured mean hip-to-table distance was $117 \mathrm{~mm}$ (79 to 142). Therefore, the mean hip-to-cassette distance was calculated as $192 \mathrm{~mm}$ (154 to 217). Trigonometry suggests that a disc of $37.17 \mathrm{~mm}$ placed on the cassette will appear as a sphere of $30 \mathrm{~mm}$ placed at the centre of the hip. This equates to a magnification of $124 \%$ (Fig. 2).

Before committing ourselves to the manufacture of customised discs, we performed a trial using readily available $2 \mathrm{~mm}$ thick mild steel discs of $36.7 \mathrm{~mm}$ diameter. We predicted that these would generate a small degree of overmagnification $(1.5 \%$ or $0.45 \mathrm{~mm}$ in a $30 \mathrm{~mm}$ sphere).

Accuracy, reliability and reproducibility were assessed on 20 patients at three-month follow-up after hip replacement. Post-operative radiographs were not used as these were routinely carried out in the patient's bed. The three-month radiographs were performed with the $30 \mathrm{~mm}$ sphere in a disposable glove between the patient's legs, and the $36.7 \mathrm{~mm}$ disc on the cassette. In order to eliminate the error from variable deformity of the foam, the table mattress was removed and the focus-to-film distance set at $1000 \mathrm{~mm}$ in all cases (Fig. 3). The images were processed using the Mdesk software. Two separate calibration procedures were 


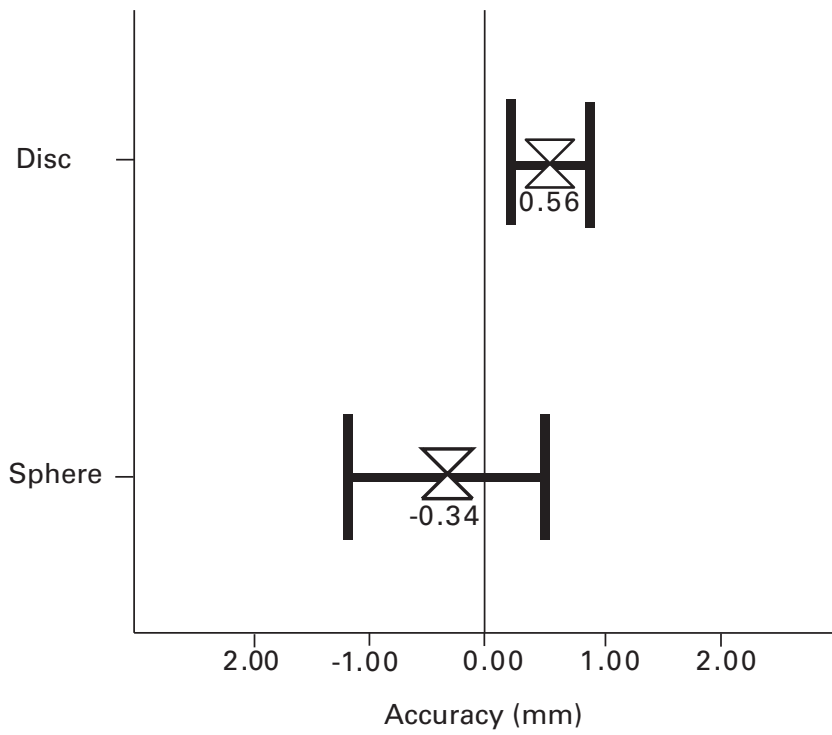

Fig. 3

Graph showing accuracy of calibration using sphere and discs with $95 \%$ confidence intervals.

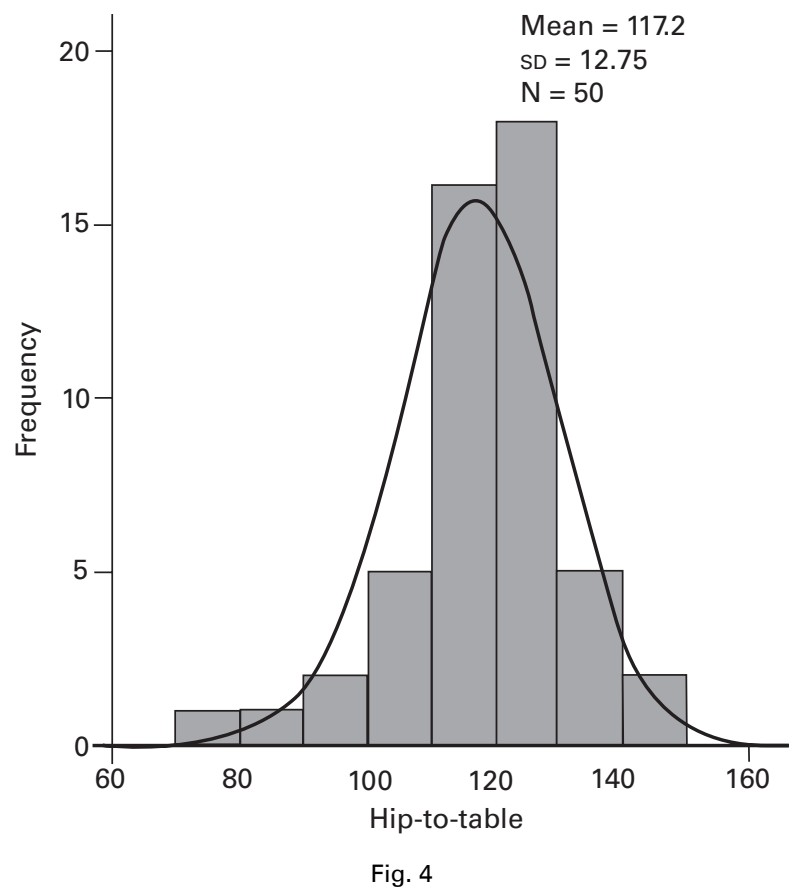

Graph showing distribution of hip-to-table distance.

performed and the size of thehead of the femoral component was measured by recording the maximum diameter of the head on three occasions. The difference between the known and observed value was recorded for the disc sphere calibrated images.

Statistical analysis. The data was analysed using SPSS v 12.0 software (SPSS Inc., Chicago, Illinois). Descriptive statistics were performed. Reliability was determined by assessing the variance of error between the disc and ball using Levene's non-parametric test. A p-value less than 0.05 was considered significant.

\section{Results}

The results of the first study showed a mean hip-to-table distance of $117.2 \mathrm{~mm}$ (79 to 142) with a distribution approximating normal (SD $12.75 \mathrm{~mm}$; Fig. 4). The accuracy of the sphere method produced a mean difference between the actual and measured head size of $-0.34 \mathrm{~mm}$ $(-5$ to 2.25$)$. The disc method produced a mean difference of $0.56 \mathrm{~mm}$ (- to 2) (Fig. 4). Levene's test suggested a significant difference between the two data sets $(\mathrm{p}=0.001)$, indicating that the disc method was more reproducible. Although both methods were found to size the femoral head accurately, the disc method proved more consistent with least deviation from true size.

This confirmed that the concept could work and precision discs were manufactured. Further calibration testing of 20 post-operative radiographs using the disc of appropriate diameter was accurate in all patients. In every case the size of the head was determined correctly by the disc calibrated image, within the $1 \mathrm{~mm}$ resolution accuracy of the Mdesk software.

\section{Discussion}

Templating and implant selection involves multiple factors, many subjective, regarding perception of best fit. We therefore did not compare the reproducibility of implant selection between the sphere and disc.

In the first pilot study, the accuracy of the disc method appeared to be inferior to the sphere method, with a mean difference of $+0.56 \mathrm{~mm}(-1$ to +2$)$ compared with $-.34 \mathrm{~mm}(-5$ to +2.25$)$. However, the use of a smaller than required disc predicted the method would over estimate implant head size by $1.5 \%$. This magnification of a $28 \mathrm{~mm}$ femoral head produces an absolute over estimate of $0.42 \mathrm{~mm}$ (remarkably close to the $+0.56 \mathrm{~mm}$ over estimation observed in the pilot study). When the predicted correct disc size was used in the second 20 patient group, absolute accuracy was found in all patients.

Of greater importance was the range and SD of the observations with the disc method being much more reproducible. This is explained by the difficulty in placing and holding a calibration object at the correct height. On occasions, the disc and sphere on the three-month images were of clearly different sizes, indicating that the sphere was not placed correctly (Fig. 5). On inspection of the images, the patient with undersizing of $-5 \mathrm{~mm}(20 \%$ undersized), had let the sphere slip onto the table. This was not detected by the radiographers, despite their experience with this technique.

Although we used a single table type for all cases, table thickness varies between manufacturers and this should be 


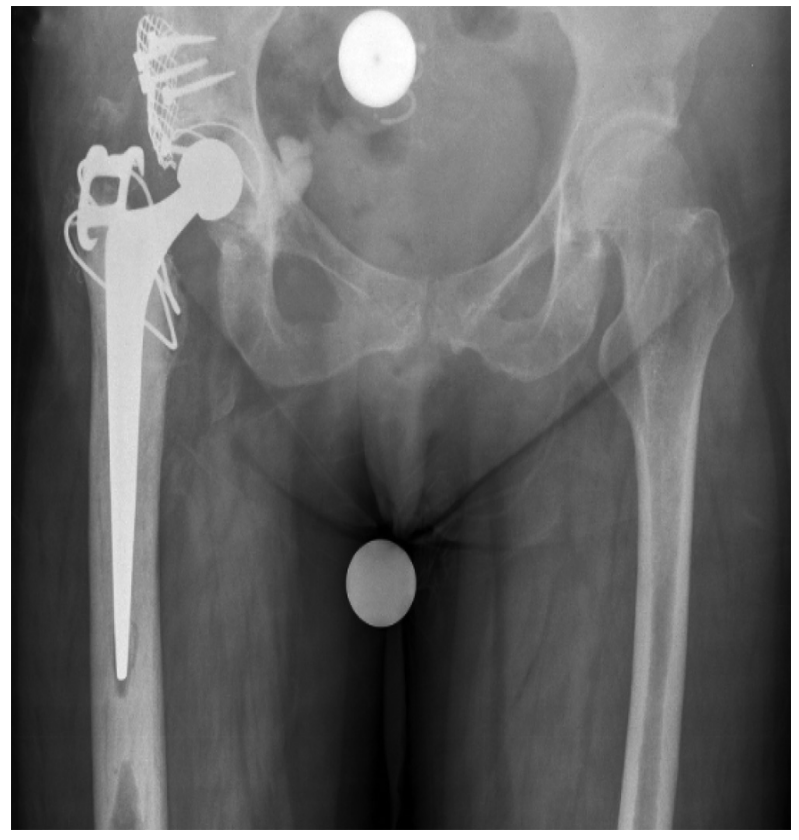

Fig. 5

Digital radiograph of the pelvis. The sphere between the legs has dropped to the table and is thereby smaller than the disc on the cassette.

considered in the initial calculations and disc selection. The mattress should be removed.

Our assessment of hip-to-table distance provides a mean distance upon which subsequent calculations are based. We were concerned that the range of hip-to-table distances would require numerous disc sizes to accommodate all patients accurately. Calculations based on larger and smaller patients and subsequent magnification errors showed that a single disc based on a hip-to-table distance of $117 \mathrm{~mm}$ would accurately size $80 \%$ of patients within an accuracy of SD $2.5 \%$. As hip prostheses increase in approximately $4 \%$ increments $(2 \mathrm{~mm}$ increment for $50 \mathrm{~mm}$ acetabular component), an accuracy of SD $2.5 \%$ was considered acceptable, and the use of a single disc justified. The remaining $20 \%$ of patients who are too big or too small are difficult to define using body measurements. It is unreasonable to expect a busy radiographer to calculate the body mass index. We therefore suggested that the radiographer makes a cursory assessment of body morphology and selects an alternative disc accordingly. Three of the second 20 post-operative patients required these alternative discs (two large and one small). We therefore have three disc sizes, 'medium', which will accurately size $80 \%$ of patients, small, for those who look strikingly thin and 'large', for those who appear significantly obese. This clearly introduces a potential source of error but the compromisewas necessary to make the technique acceptable to our radiographers and it does not appear to affect accuracy.

We have shown our technique to be accurate, robust, reliable, acceptable to patients and radiographers and cost effective. We have commissioned a large number of custom manufactured $2 \mathrm{~mm}$ thick stainless steel discs of the precise diameter for each table across many hospitals in our area. The cost per disc is approximately $£ 2.50$. Further development may include incorporation of a calibration marker within the cassette. However, for the moment, the ritual of placing the disc on the cassette reminds the radiographer about other details such as setting the film-focus distance accurately and removing the mattress.

In conclusion, we have shown that a calibration technique requiring estimation of the centre of the hip and placement and retention of an out-of-plane calibration object is unreliable. Despite potential inter-patient errors our disc method appears to be more accurate and reliable. The expensive practice of printing radiographs and subsequent inaccurate pre-operative templating using acetates can be abandoned.

No benefits in any form have been received or will be received from a commercial party related directly or indirectly to the subject of this article.

\section{References}

1. Edwards M. Department of Health PACS Implementation. http://www.dh.gov.uk/ en/Publicationsandstatistics/Lettersandcirculars/Dearcolleagueletters/ DH_4104114 (date last accessed 18 September 2007).

2. Della Valle AG, Padgett DE, Salvati EA. Preoperative planning for primary total hip arthroplasty. J Am Acad Orthop Surg 2005;13:455-62.

3. The B, Diercks RL, van Oojen PM, van Horn JR. Comparison of analog and digital preoperative planning in total hip and knee arthroplasties: a prospective study of 173 hips and 65 total knees. Acta Orthop 2005;76:78-81.

4. White SP, Shardlow DL. Effect of introduction of digital radiographic techniques on pre-operative templating in orthopaedic practice. Ann $R$ Coll Surg Engl 2005;87:53-4.

5. Conn KS, Clarke MT, Hallett JP. A simple guide to determine the magnification of radiographs and to improve accuracy of preoperative templating. J Bone Joint Surg [Br] 2002;84-B:269-72.

6. Oddy MJ, Jones MJ, Pendegrass CJ, et al. Assessment of reproducibility and accuracy in templating hybrid total hip arthroplasty using digital radiographs. $J$ Bone Joint Surg [Br] 2006;88-B:581-5.

7. Wimsey S, Pickard R, Shaw G. Accurate scaling of digital radiographs of the pelvis: a prospective trial of two methods. J Bone Joint Surg [Br] 2006;88-B:1508-12. 